\title{
Atypical Central Serous Chorioretinopathy with Bilateral Bullous Exudative Retinal Detachment Following Systemic Corticosteroid Therapy
}

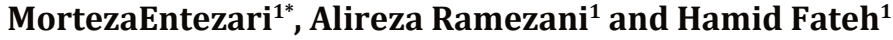 \\ ${ }^{1}$ Department of Ophthalmology, Ophthalmic Research Center, Imam Hossein Medical Center, Shahid Beheshti Medical Sciences, Tehran, Iran
}

Received: 11 November, 2016; Accepted: 28 Febraury, 2017 ; Published: 10 March, 2017

*Corresponding author: Morteza Entezari, Professor, Department of Ophthalmology Ophthalmic Research Center, Imam Hossein Medical Center, Shahid Beheshti Medical Sciences, Tehran, Iran. Tel: +98 21 22591616, Fax: +98 21 22256213; E-mail: entmort@hotmail.com

\begin{abstract}
Purpose: To report a case of an atypical idiopathic central serous chorioretinopathy (ICSC) with bilateral retinal detachment following intravenous high-dose corticosteroid therapy.

Methods: A 24 year-old girl presented with sudden painless visual loss in both eyes. Complete ophthalmic examination, fluorescein angiography (FA), indocyanin green angiography (ICG), and optical coherence tomography (OCT) were done and patient was followed for six months.

Results: Loss of vision in her right eye and progression of visual loss in left eye were developed after intra-venous steroid therapy. Best corrected visual acuity (BCVA) was $1 / 10$ and $2 / 10$ in her right and left eyes, respectively. In funduscopic examination, we found bullous exudative retinal detachment in her both eyes without vitreous reaction. Fundus fluorescein angiography (FA) revealed diffuse leakage and indocyanin green angiography (ICG) showed evidence of diffuse choroidopathy. Optical coherence tomography revealed multiple neurosensory and pigment retinal detachments. The patient was followed with impression of atypical bilateral ICSC without any treatment. Visual acuity improved and retinal attachment occurred in both eyes during 6 months.
\end{abstract}

Conclusions: Diagnosis of atypical ICSC and role of corticosteroids on development of ICSC must be considered in this special cases with bilateral bullous retinal detachment.

Keywords: OCT; Atypical Central Serous Chorioretinopathy; Corticosteroids

\section{Introduction}

Idiopatic Central Serous Chorioretinopathy (ICSC) is a disease in which a serous detachment of the neurosensory retina occurs over an area of leakage from the choriocapillaris through the retinal pigment epithelium (RPE) causing a circumscribed area of retinal detachment. In most cases, retinal detachment resolves spontaneously with a good visual recovery [1].

On rare occasions, multiple retinal neurosensory detachments, bullous detachments and bilateral involvements are among the atypical forms of ICSC presentations [2-4]. This atypical presentation following systemic corticosteroid therapy may be complicate diagnosis of ICSC $[5,6]$.

In this report, we present a young lady who was taking corticosteroids after an incorrect diagnosis and developed atypical bilateral CSC characterized by diffuse serous retinal detachment.

\section{Case History}

A 24-year-old female referred to our clinic with complain of bilateral painless visual loss of both eyes since 15 days ago. In another center, she had received an intravenous steroid pulse therapy for 3 days followed by oral prednisolone with the diagnosis of optic neuritis for her visual decrease in the left eye. Vision loss in her right eye developed after initiation of the steroid therapy.

Best corrected visual acuity was $1 / 10$ and $2 / 10$ in her right and left eyes, respectively. There was no afferent pupillary defect. Anterior segment examination by slit lamp bio microscopy was unremarkable for both eyes. No inflammatory cell was detected in anterior chamber and vitreous cavity of either eye. Intraocular pressures were normal. Funduscopic examination revealed multiple circumscribed areas of retinal neurosensory detachment in both eyes.

Fluorescein Angiography (FA), indocyanin green angiography (ICG) and optical coherence tomography (OCT) were performed for the patient with the clinical impression of atypical CSC. FA showed multiple ill-defined hyperfluorescent foci showing increasing size in the late frames of angiograms. In OCT, multiple neurosensory and pigment epithelial detachments in the posterior pole of both eyes were revealed. ICG demonstrated a diffuse leakage from choriocapillaris (Figure 1). 

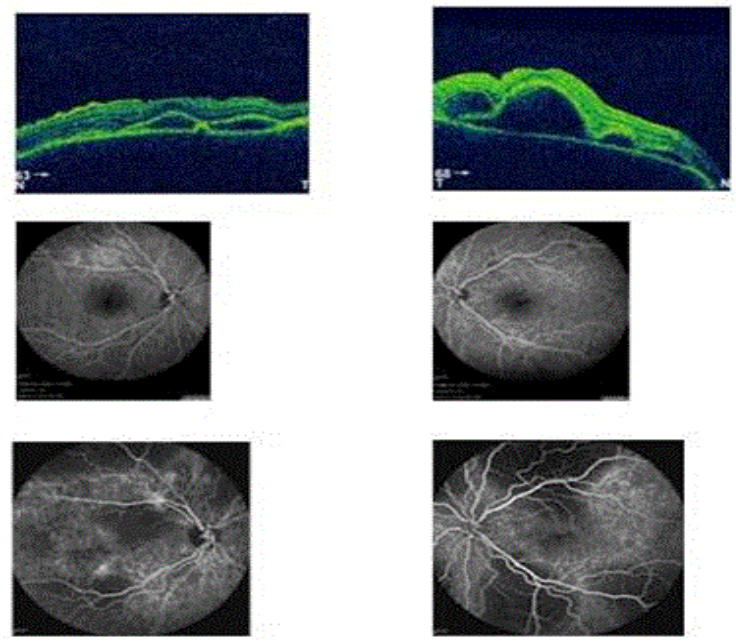

Figure 1: OCT of right (a) and left (b) eyes: Cystoid hyporeflective spaces compatible with retinal neurosensory and pigment epithelium detachments. Mid arteriovenous phase of FA of right (c) and left (d) eyes show Multiple areas of hypo-fluorescence and pinpoint hyper-fluorescence. Fundus ICG of right (e) and left (f) eyes reveal hypo-fluorescence and pin point hyper-fluorescence areas of choriodal leakages

With the diagnosis of CSC aggravated by steroid, the patient was asked to discontinue the oral prednisolone and was followed with no further treatment. After 6 weeks, corrected visual acuity improved to $6 / 10$ and $7 / 10$ in her right and left eyes, respectively and a great regression of the neurosensory detachments was noticed on optical coherence tomography pictures. After 6 months, visual function was restored to normal and the patient achieved a 10/10 vision in her both eyes. The retina attached completely with improvement of leakage in FA (Figure 2).
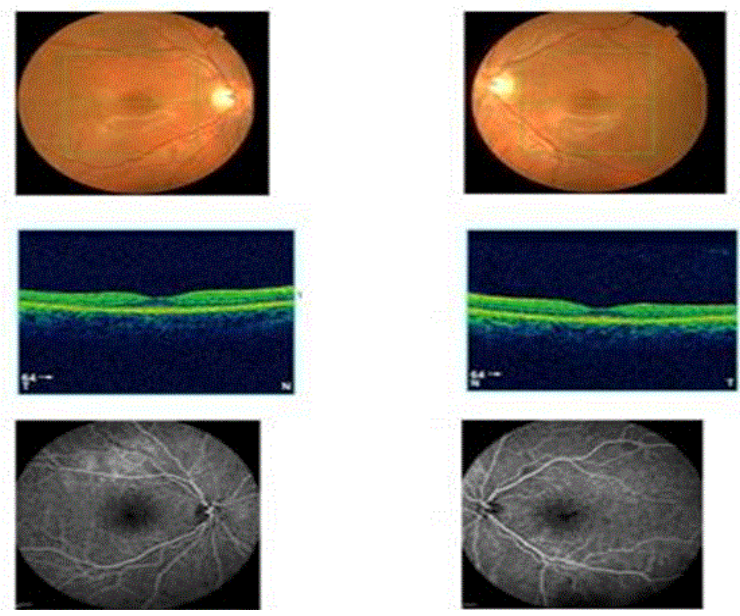

Figure 2: Color Fundus photogarphs of right (a) and left (b) eyes, 6 months after first examination. OCT of right (c) and left (d) eyes show no retinal and subretinal fluid accumulation. FA of right (e) and left (f) eyes after 6 months

\section{Discussion}

Patients receiving any drug form steroids are at risk for development of CSC and or even exacerbation of a reexisting CSC into a form of atypical CSC sometimes with bilateral involvement [7]. This has been reported by Wessing et al as an increase in the subretinal fluid in patients under systemic steroid treatment [8]. Gass et al has also shown that glucocorticoids used for the treatment of CSC may intensify the condition [6].

Our patient had an exacerbation of the CSC in one eye and development of a severe form of CSC in the fellow eye following receiving steroid therapy for an incorrect diagnosis. At presentation, we noticed bollous retinal detachments in her both eyes. Such an atypical presentation of CSC with bilateral bullous exudative retinal detachment is confusing and challenging in terms of diagnosis. However, a careful history taking is very helpful since this presentation is more prevalent in patients receiving exogenous corticosteroids, although spontaneous cases have also been reported $[9,10]$.

This variant of CSC is a diagnostic dilemma and must be distinguished from rhegmatogenous retinal detachment and other forms of serous retinal detachments. A careful ophthalmic examination may be helpful in establishment a correct diagnosis. Absence of vitreous cells, presence of yellow-white lesions with single or multiple pigment epithelial detachments, shifting fluid with fluctuating vision, and absence of retinal break may lead us to differentiate it from other similar diseases. In addition, FA and ICG as well as OCT are essential ancillary tests to confirm the diagnosis [3].

Our case presented with bilateral bullous serous retinal detachment with no vitreous cell following high dose steroid. Similar presentations have also been reported previously $[3,5,7]$. In some of these reports however, subretinal fibrosis was developed in the course of the disease $[3,5]$. Nonetheless, our patient improved completely over a period of 6 months without any apparent subretinal fibrosis.

\section{Conclusion}

In conclusion, correct diagnosis of CSC avoids imposing inappropriate treatments on patients. Corticosteroids usage with diagnosis of optic neuritis is a common mismanagement that may worsen the condition. Atypical CSC must be considered as a differential diagnosis in any patient with bullous exudative retinal detachment without vitreous involvement receiving corticosteroid.

\section{References}

1. Mazzuca DE, Benson WE. Central serous retinopathy: variant. Surv Ophthalmol. 1986;31(3):170-174

2. Brancato R, Bandello F. Central serous retinopathy (atypical forms). Bull Soc Belge Ophtalmol. 1991;240:119-131

3. Sahu DK, Namperumalsamy P, Hilton GF, de Sousa NF. Bullous variant of Idiopathic central serous chorioretinopathy. Br J ophthalmol. 2000;84(5):485-492. doi: 10.1136/bjo.84.5.485

4. Gass JDM. Stereoscopic Atlas of Macular Diseases. St Louis, CV Mosby Co. 1997,52-70. 
5. Bouzas EA, Karadimas P, Pournaras CJ. Central Serous Chorioretinopathy and Glucocorticoids. Survey of Ophthalmology. 2002;47(5):431-448

6. Gass JD, Little H. Bilateral bullous exudative retinal detachment complicating idiopathic central serous chorioretinopathy during systemic corticosteroid therapy. Ophthalmology. 1995;102(5):737747

7. Yoon IS, Do SJ, Im SJ. A case of Atypical Central Serous Chorioretinopathy with Bullous Retinal Detachment. J Korean Ophthalmol Soc. 1991;32(11):997-1002
8. Wessing A. Changing concept of central serous retinopathy and its treatment. Trans Am Acad Ophthalmol Otolaryngol. 1973;77(3): OP275-80

9. Arndt C, Sari A, Ferre M, Parrat E, Courtas D, De Seze J, et al. Electrophysiological effects of corticosteroids on the retinal pigment epithelium. Invest Ophthalmol Vis Sci. 2001;42(2):472-475

10. Matsuo T, Nakayama T, Koyama T, Matsuo N. Multifocal pigment epithelial damages with serous retinal detachment in systemic lupus erythematosus. Ophthalmologica. 1987;195(2):97-102 